\title{
PRIMARY AMYLOIDOSIS
}

\author{
G. Patrassi, M.D. \\ Clinica Medica Generale, University of Padua, Italy \\ (Director: Professor G. Patrassi)
}

AMONG the changes in human pathology due to the therapeutic advances of recent years must be numbered the almost complete disappearance of cases of secondary amyloidosis. This is almost certainly due to the much lower incidence of some of the determining factors of this condition (chronic tuberculosis, prolonged suppuration). In contrast, however, primary amyloidosis has become far more prevalent.

Primary amyloidosis was very rare until some twenty years ago (Whittlesey recorded 70 cases in 1950), whereas nowadays it is a well-known if infrequent condition. But in order to be in a position to diagnose it, it is essential to bear its existence in mind and to be aware of its fundamental clinical signs. In this way, having been fortunate to study a particular case in 1946, I have since been able personally to collect a further five cases.

There can be no doubt that organ needlebiopsy, particularly of the liver and kidney, has made a major contribution to the diagnosis of this condition in vivo.

If the diagnosis of primary amyloidosis is to be exact, it is essential that the patient's medical history should reveal no chronic debilitating disease of the type which is known to account for secondary amyloidosis. It is however possible that a major though uncharacteristic disease occurring at some time in the past medical history of the patient may be relevant. (One of our patients, for instance, had suffered in the past from a prolonged specific peritonitis, which, however, had since completely healed). This would seem to suggest that primary amyloidosis might commence in the course of an illness, even if that illness itself clears up completely.

The clinical and morbid anatomical data for primary amyloidosis do not differ greatly from those of secondary amyloidosis, with the exception of the underlying illness in the latter.

Nevertheless, I should like to point out that in my experience hepatomegaly is the most important finding in the clinical picture of primary amyloidosis. The hepatomegaly is both marked and progressive: at times it is extreme, the liver being of hard or even woody consistency, with a coarse and pain-free edge. Liver function tests give equivocal evidence of a varying and inconstant nature during the different stages of the illness.

This enlargement of the liver may persist for many years without causing any symptoms, only rarely giving rise to jaundice or portal hypertension. It perhaps goes without saying that confusion with an hepatic neoplasm is the commonest error in diagnosis, as happened in two of my own cases. On the other hand, in the first case of my series, a concomitant hypoglycæmia led me to believe that I was dealing with a glycogenic hepatomegaly of the von Gierke type (Fig. 1).

The spleen-following "big brother", the liver-also increases in size, though this appears to be of no particular significance.

The second important clinical finding is a renal disturbance, taking the form of a nephrotic syndrome with marked proteinuria. It is this which frequently gives the clue to the correct diagnosis as its appearance calls for a needle biopsy of the kidney (Fig. 2).

The blood picture does not contribute much to the diagnosis because the increase in the number of white cells, which frequently occurs, suggests the erroneous diagnosis of carcinoma of the liver. Of far greater significance is the marked increase in the number of platelets in the circulation; a finding which I have noted in a number of my cases.

I do not intend to deal here with those other clinical factors which are so well known in cases of secondary amyloidosis, such as myocardial amyloid deposits (causing disturbances of conduction, low ECG voltages and signs of progressive heart failure), the formation of amyloid deposits in the mediastinum, changes in the skin and peripheral nerves, macroglossia, etc.

I should however like to point out that although it is usually extremely difficult to diagnose primary amyloidosis, this may be 

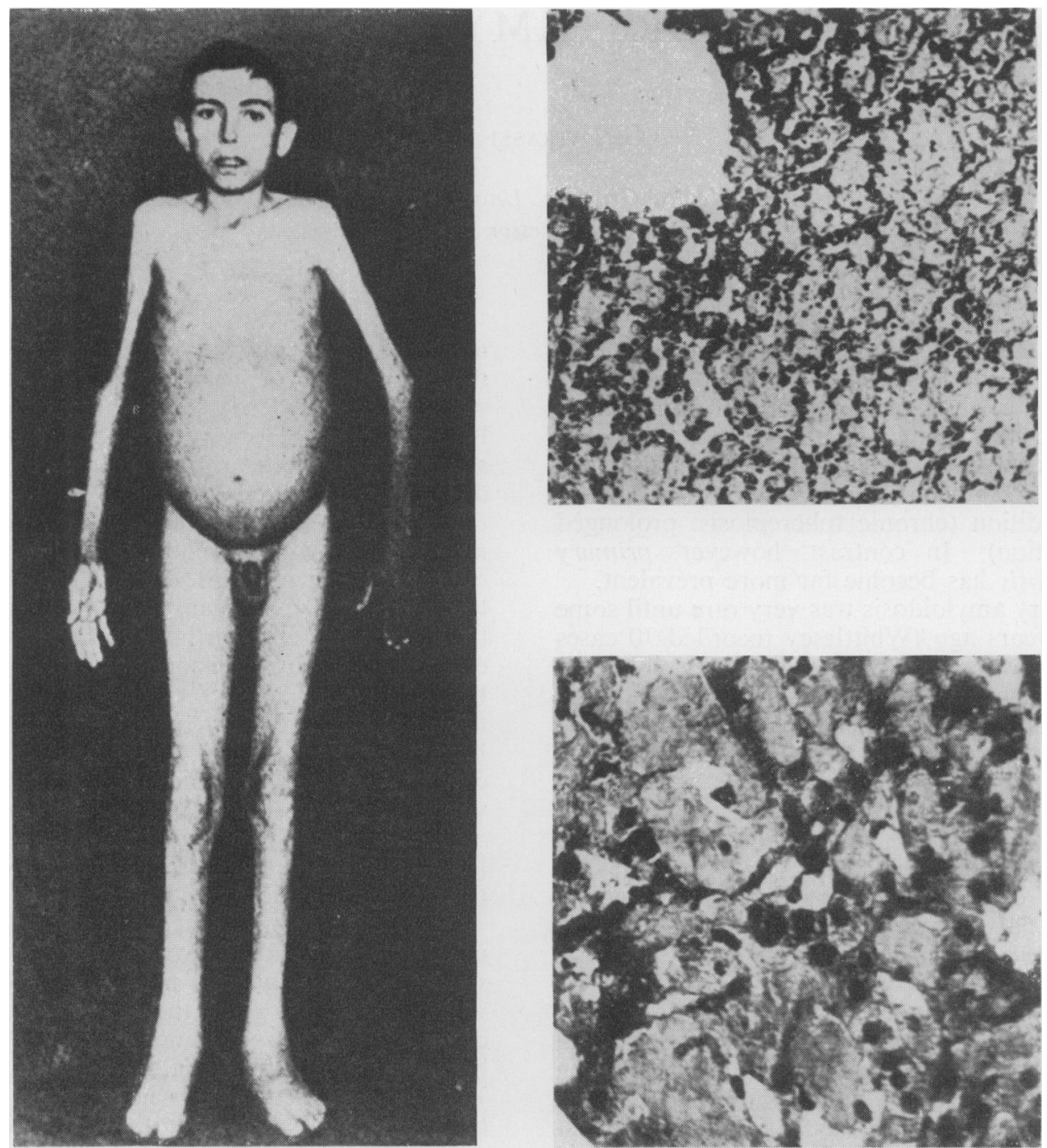

FIG. 1.-Case of primary amyloidosis in an underdeveloped (male, aged 18) subject with enormous hepatomegaly and high grade ascitis. The correct diagnosis was possible only histologically (see right) by means of a surgical biopsy of the liver. Before that the clinical signs suggested von Gierke's disease.

suspected when any of the above mentioned signs is superimposed on an already existing, basic clinical picture of debility, fever, loss of strength, lack of appetite, and a high blood sedimentation rate. But above all on finding the combination of gross hepatomegaly with the nephrotic syndrome.

I have already indicated the importance of organ needle-biopsy in diagnosis. But I would add the warning that this procedure calls for very special care in view of the solid consistency of the organs to be subjected to biopsy, and in particular that of the liver and spleen, where such a manoeuvre may lead to prolonged ands therefore dangerous bleeding. It was for thisu reason that $I$ felt it prudent to opt for surgicaR rather than needle biopsy on two of my patients ${ }_{\sigma}^{\omega}$

Moreover, cases of spontaneous rupture of the spleen (Drapiewski, 1955) as well ast traumatic rupture (King and Oppenheimer@ 1948) have been known to occur.

In one of our cases, it was found necessaryo to remove the spleen in order to stop the bleeding which followed a splenoportography $\stackrel{\mathbb{D}}{\mathbb{D}}$ 

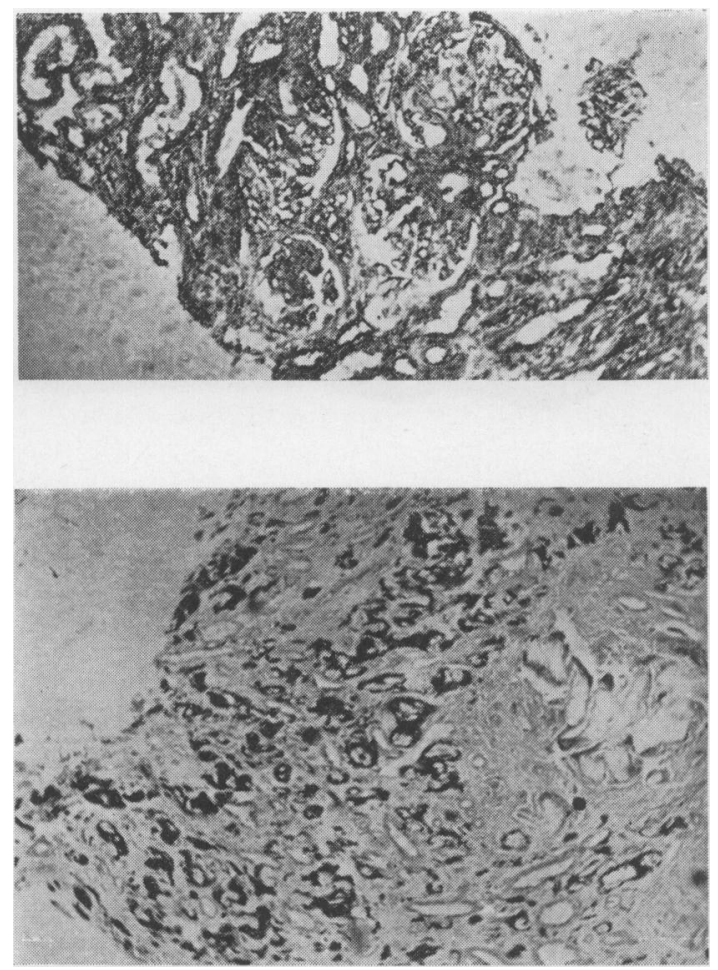

FIG. 2.-Needle biopsy of the kidney: amyloidosis of the cortical substance and particularly of the glomeruli (above); amyloidosis of the medulla (below).

Unfortunately the patient subsequently suffered a peritonitis secondary to an amyloid appendicitis and died post-operatively of a pulmonary embolism.

From the point of view of diagnosis it is important to make a study of plasma proteins using electrophoresis. This is characterised by a hypoproteinæmia sometimes accompanied by an increase in gamma-globulin or alpha fraction, in the latter case either in lipoproteins or glycoproteins (Fig. 3).

This hypoproteinæmia is normally attributed to the accompanying nephrotic syndrome. Nevertheless, I would point out that in one of my own cases (a student who is still alive and has been under surveillance for many years) there was noted a considerable increase in the alpha-2 and beta-globulins despite the absence of any obvious renal disturbance.

Undoubtedly, the fundamental biochemical change in both primary and secondary amyloidosis is a profound disturbance of protein metabolism.
This theory is confirmed not only by the biochemical findings outlined above but also by the fact that the amyloid substance is a glycoprotein in which a number of polysaccharide groups are bound to the globulin nucleus.

Although research undertaken with a view to showing the existence of a "pre-amyloid body" in the blood stream has not yet yielded any reliable results, there are certain facts which would seem to confirm that - even though the precipitation of amyloid in the tissues may be provoked to a certain extent by local lesions-it is to the changes in blood protein that we must look for the fundamental cause. And these facts are:

1. Experimentally produced amyloidosis (from Kuczinski onwards) has been achieved by a high protein diet;

2. Plasmocytoma is an illness in which hyperproteinæmia is frequently the determining cause of an atypical amyloidosis;

3. The illnesses which cause secondary amyloidosis are characterised by a constant hyperglobulinæmia.

To sum up therefore, there are grounds for a theory that the pathogenesis of primary amyloidosis (which is not unlike that of secondary amyloidosis) is linked to a primary disturbance of blood protein.

A further factor, which may be bound up with that outlined above, is that primary amyloidosis may show a familial pattern.

It is therefore a legitimate assumption that the disturbance in blood protein responsible for primary amyloidosis may be familial as it is in analbuminæmia and agammaglobulinæmia.

It is well known that the "rheumatoid factor" has been detected in the healthy relatives of patients suffering from rheumatoid arthritis.

Thus it may be inferred that in some families there exists a constitutional blood protein abnormality which in the presence of amyloidproducing factors and of tissue factors results in the precipitation of amyloid in susceptible organs and organelles.

It is no easy task to define just exactly what this really is. Nevertheless, it should be pointed out that in some cases-as already statedthere is a more or less marked increase in the alpha-2 and beta-globulin content; according to Andolfatto Zaglia (1963), the immuno-electrophoretic identification of a marked increase in the alpha-2 $\mathrm{M}$ macroglycoprotein fraction (even when renal pathology is absent) is characteristic 


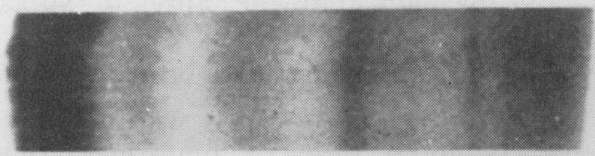

Primary

Amyloidosis

G. G., male, age 29

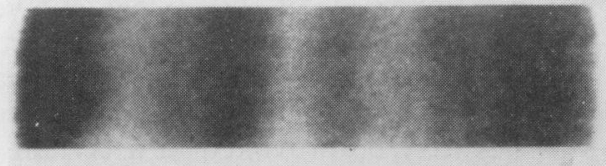

\section{Primary \\ Amyloidosis \\ C. I., female, age 50}

Primary

Amyloidosis

A. E., female, age 60

\section{Secondary}

Amyl. TBC.

B.V. male

age 46

Secondary

Amyl. TBC.

B.A. female age 48 .
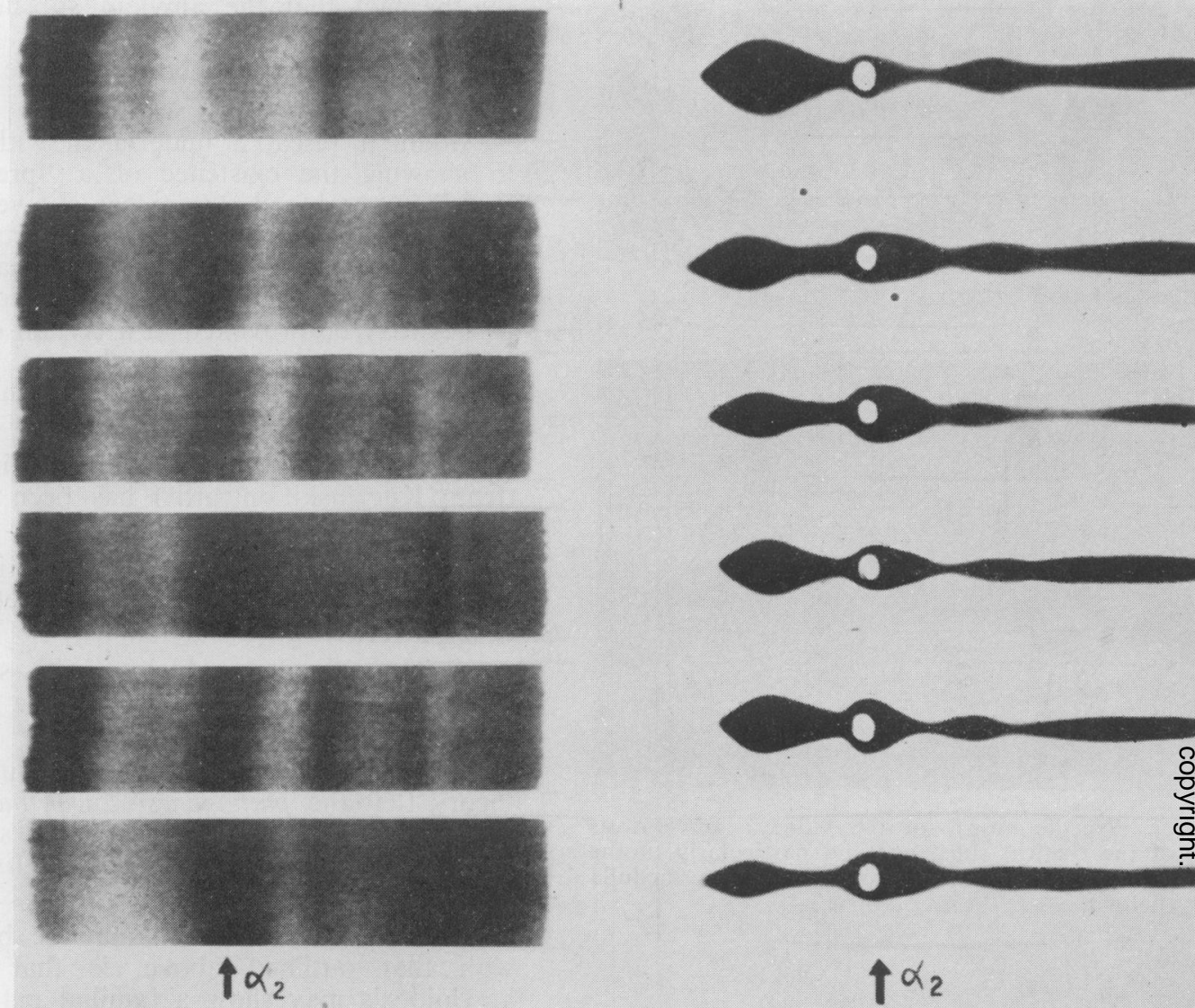

Fig. 3.-Both paper and agar-gel electrophoresis show a constant increase of the $\alpha_{2}$ fractions in 3 cases of primary amyloidosis and in 2 cases of secondary amyloidosis. Above, the normal pattern.

of primary amyloidosis and of secondary amyloidosis caused by tuberculosis (it does not occur in myelomatous para-amyloidosis (Fig.4)). All this would seem to indicate that the unknown substance which precipitates in the tissues is in some way allied to the glycoproteins.

Further research must be conducted with a view to discovering which of the organs concerned in protein synthesis is directly responsible for this potential or actual "dysproteinæmia".

The clinical and histopathological pictures point to the liver as being the primary factor.

In fact in many cases in the literature-and in all of mine - the liver shows changes at a very early stage; at times the hepatomegaly is of such dimension as to make one think that the process of protein storage finds here largest and most imposing hoard (Fig. 5).

On the other hand, the histological picture makes it possible to identify the gradual for mation of the first amyloid deposits between the liver cell and the Kupfer cell.

It should be remembered that although the liver is largely responsible for the formation of albumins, it also plays an important part the formation of some globulins (alpha and beta rather than gamma) which are so closecy connected with lipoid and glycoid metabolism.

All this would seem to suggest that the liver may well be the first and most important element in the disturbance of protein metsbolism and effects the introduction of the 
IMMUNOELECTROPHORETIC PATTERNS WITH ANTISERA
Pasteur 223
(anti $\mathrm{A}, \alpha, \beta$, and $\gamma$ glob.)
Pasteur 306
$\alpha$ and $\beta_{2} \mathrm{~A}$ glob.)
Behringwerke
$\stackrel{2}{\gtrless}$
(specific anti $\alpha_{2} \mathrm{M}$ glo

Normal

Human

Serum

\section{Primary}

Amyloidosis G. G., male age 29

Primary

Amyloidosis

C. I., female age 50

Secondary

Amyl. TBC.

B.A. female age 48 .
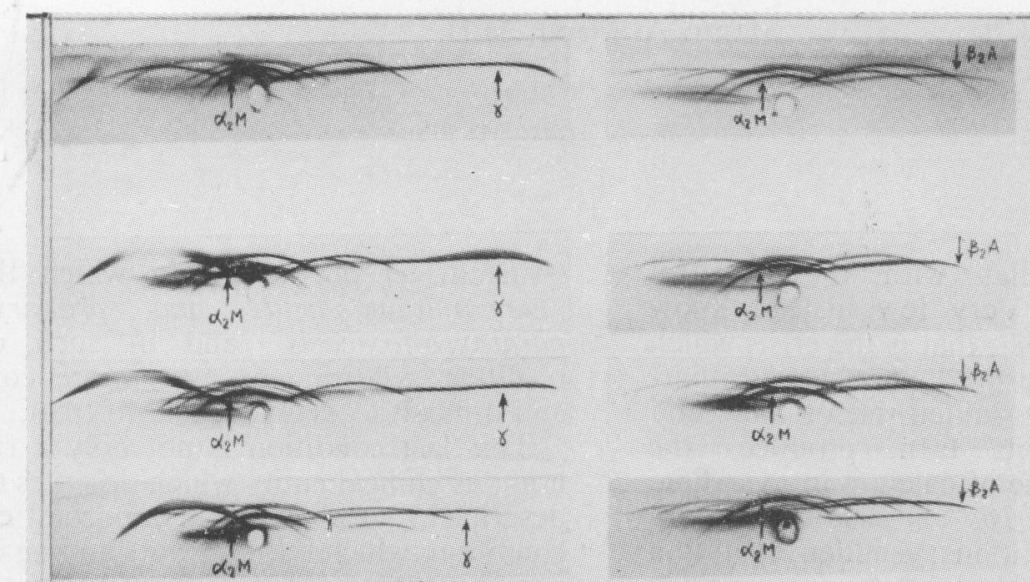

Fig. 4.- In the sera of amyloidotic patients there is a remarkable increase of precipitating reactions of $\alpha_{2} \mathrm{M}$ macroglycoproteins (reference left and middle column). These reactions also occur with a specific antiserum (reference right column).

There is also a constant increase of precipitating reactions of $\beta_{2} \mathrm{~A}$ immunoglobulins (reference middle column).

The reactions of the other fractions, especially of $\gamma$ globulins (reference left column), are not constant.

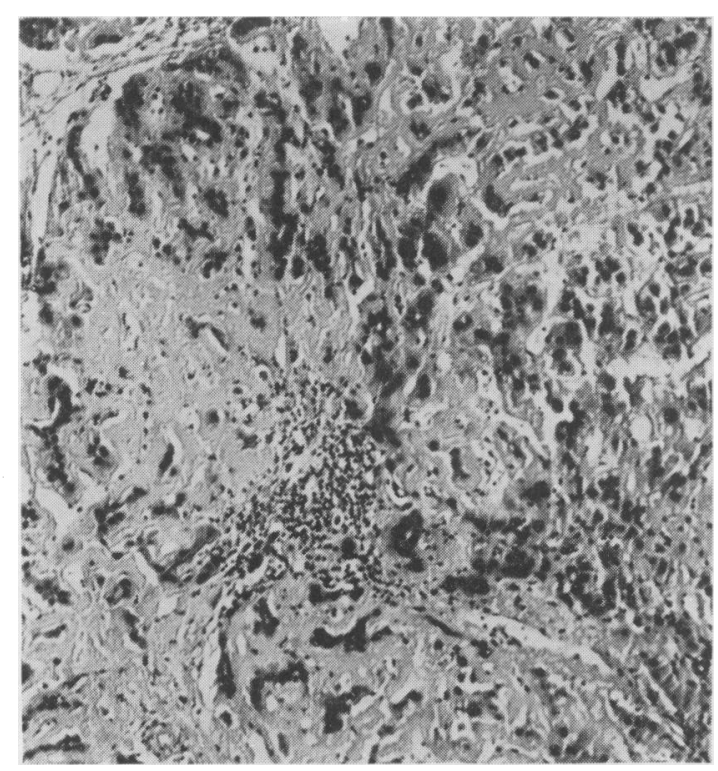

FIG. 5.-Needle biopsy of the liver. The liver cells are compressed by the amyloid substance and become atrophic. Note the rich reactive cellular infiltration. abnormal glycoproteins into the circulation, later to be absorbed by the musculomesenchymal or connective-vascular tissues of such other organs as the kidneys, pancreas, adrenals and muscles.

\section{REFERENCES}

Andolfatto-Zaglia, G. (1963): Aspetti Sierologici e Fattori Autoaggressivi nella Patogenesi dell' Amiloidosi, Acta med. patav., 23, 173.

DraPIEWSKI, F. J. (1955): Primary Amyloidosis with Spontaneous Rupture of the Spleen and Sudden Death, Ann. intern. Med., 43, 406.

KING, F. H., and OPPENHEIMER, G. D. (1948): Rupture of Amyloid Spleen, ibid, 29, 374

Patrassi, G. (1946): La Tesaurismosi Proteica (su di un Caso di Amiloidosi Pluriviscerale sine Causa, con Enorme Epatomegalia), Acta med. patav., 7, 1.

Whittlesey, W. N. (1950): Primary Atypical Amyloidosis, Arch. intern. Med., 86, 245. 